Check for updates

Cite this: RSC Adv., 2017, 7, 30862

\title{
Molecular-level insight into the binding of arginine to a zwitterionic Langmuir monolayer $\dagger$
}

\author{
Joaquín Klug, ${ }^{\star a b}$ Diego Masone ${ }^{a}$ and Mario G. Del Pópolo (iD ab
}

Solutions of the cationic amino-acid arginine $\left(\mathrm{Arg}^{+}\right)$in contact with a phospholipid monolayer are investigated by molecular dynamics simulations. The results show that $\mathrm{Arg}^{+}$binds strongly to the lipid/ water interface, with adsorption free-energies ranging from -43.8 to $-22.2 \mathrm{~kJ} \mathrm{~mol}^{-1}$, depending on the amino-acids concentration. The large binding energies are attributed to hydrogen bonding between the charged moieties $\mathrm{Arg}^{+}$and the phosphate and carbonyl groups of the phospholipids, that compensate for changes in the levels of hydration upon adsorption. We show that a concentrated layer of $\mathrm{Arg}^{+}$, tightly bound to the interface, has little effect on the compression isotherm and the lateral mechanical properties of the monolayer, while having a substantial impact on the interfacial electrostatic potential and the lateral mobility of the lipids. These effects are readily explained in terms of the arrangement that the amino-acids adopt when bound to the monolayer.

Received 12th May 2017

Accepted 8th June 2017

DOI: $10.1039 / \mathrm{c} 7 \mathrm{ra05359b}$

rsc.li/rsc-advances compounds, ${ }^{16,17}$ and anti-cancer drugs. ${ }^{18,19}$ Unfortunately, the standard measurements performed on typical monolayer experiments do not deliver molecular-level insight into the structure and dynamics of these systems. Nor do they allow the decomposition of the binding energy of adsorbates into different contributions, or the identification of specific adsorbate-lipid interactions. The lattes information is, in fact, valuable and could be used to optimise the response of a lipid aggregate to a particular adsorbate, for example. ${ }^{20}$

Molecular simulations provide a microscopic view of the system's behaviour that can help to interpret experimental trends and data. Molecular Dynamics (MD), in particular, has been extensively used to analyse Langmuir monolayers with atomistic $^{21-32}$ and coarse-grained ${ }^{32-40}$ levels of detail. A wide variety of amphiphilic molecules have been examined by simulations, ranging from fatty acids to detergents and lipopeptides, but undoubtedly the most popular amphiphiles have been phospholipids and their mixtures with other lipids and sterols, as the resulting monolayers have a closer resemblance to natural bio-membranes.

As discussed in more detail below, simulations have been able to reproduce experimental compression isotherms ${ }^{22,25,32,35-37,40}$ and the interfacial electrostatic potential $^{27,41-43}$ of phospholipids' monolayers with varying degrees of accuracy. Also, some studies have focused on the interaction of ions, ${ }^{44,45}$ drugs,${ }^{24}$ dyes, ${ }^{46}$ and proteins $\mathrm{s}^{23,47}$ with LM, but only a few of them have examined the effect of adsorbates on the compression isotherm ${ }^{45}$ and interfacial potential, ${ }^{24,45}$ or have discussed the adsorption mechanism in terms of a binding freeenergy profile. ${ }^{46,48}$

In the present work we look into the binding of a cationic amino-acid (arginine, $\mathrm{Arg}^{+}$) to a zwitterionic
${ }^{a}$ CONICET \& Facultad de Ciencias Exactas y Naturales, Universidad Naci
Mendoza, CP5500, Argentina. E-mail: mdelpopolo@fcen.uncu.edu.ar

${ }^{b}$ Atomistic Simulation Centre, School of Mathematics and Physics, Queen's University Belfast, University Road, Belfast BT7 1NN, Northern Ireland, UK. E-mail: $m$. del-popolo@qub.ac.uk

$\dagger$ Electronic supplementary information (ESI) available. See DOI: 10.1039/c7ra05359b 
dipalmitoylphosphatidylcholine (DPPC) monolayer. The interest in $\mathrm{Arg}^{+}$stems from the fact that it is the main component of cell-penetrating-peptides (CPP). These are highly hydrophilic polycationic peptides, that readily cross natural and artificial lipid bilayers by a mechanism which is still unknown. ${ }^{20}$ For the current purposes, it is enough to say that the strength of the binding of $\mathrm{Arg}^{+}$to the lipid surface, and possibly the associated effects on the local mechanical properties of the membrane, modulate the translocation rate. ${ }^{49,50}$ The aim of this paper is to describe how the adsorption of $\mathrm{Arg}^{+}$affects the compression isotherm of the DPPC monolayer, its interfacial electrostatic potential, and the lateral mobility of the lipids, all properties that can be accessed experimentally. We also correlate these properties with the amino-acids' adsorption free energy, which is in turn interpreted in terms of changes of hydration and specific interactions between the amino-acids' and lipids' functional groups.

\section{Computational details}

The simulated systems were made of 64 DPPC molecules forming a mono-molecular layer adsorbed on a water slab. Periodic boundary conditions were used along the $X, Y$ and $Z$ directions, the latter being perpendicular to the plane of the monolayer. The simulation box was orthorhombic, with constant $Z$-dimension $L_{z}$, while the other two dimensions $\left(L_{x}=L_{y}\right)$ were allowed to adjust in response to an imposed surface tension. Three different systems were considered: in the first case the aqueous sub-phase was made of pure water; in the other two it was a concentrated solution of $\mathrm{Arg}^{+}$and its corresponding counter-ion $\mathrm{Cl}^{-}$. The number of phospholipids, water molecules, amino-acids and chloride ions making up each system is reported in Table 1. Fig. 1 shows a representative configuration of our Langmuir monolayer configuration, together with the molecular structure of DPPC, $\mathrm{Arg}^{+}$and $\mathrm{Cl}^{-}$. The ellipses highlight the most relevant functional groups. Note that the form of arginine used in this work has three charged moieties, the guanidinium, the carboxylate and the amino groups; while only the choline and the phosphate groups of DPPC bear a whole electrical charge.

Molecular interactions were modelled combining the forcefield of Berger et al..$^{51}$ for DPPC, with the TIP4P/2005 (ref. 52) water model, as proposed and thoroughly tested by Mohammad-Aghaie et al. ${ }^{25} \mathrm{Arg}^{+}$and $\mathrm{Cl}^{-}$were described by the GROMOS-53a6 force-field. ${ }^{53}$ Long-range coulombic forces were accounted for using the particle mesh Ewald method, ${ }^{54}$ with a real space cut-off of $1.7 \mathrm{~nm}$, that was also applied to the Lennard-Jones potentials, and a Fourier spacing of $0.16 \mathrm{~nm}$. A

Table 1 Composition and $Z$-dimension, $L_{z}$, of the simulated systems. Number of DPPC, $\mathrm{Arg}^{+}, \mathrm{Cl}^{-}$, and water molecules. [ArgCl] is the molar concentration of $\mathrm{ArgCl}$

\begin{tabular}{llllll}
\hline System & $N_{\text {DPPC }}$ & $N_{\mathrm{Arg}^{+}}$ & $N_{\mathrm{w}}$ & {$[\mathrm{ArgCl}]$} & $L_{z}[\mathrm{~nm}]$ \\
\hline Pure DPPC & 64 & 0 & $\sim 4100$ & 0 & 12.2 \\
DPPC $:$ Arg $(64: 10)$ & 64 & 10 & $\sim 6600$ & 0.084 & 16.5 \\
DPPC : Arg $(64: 20)$ & 64 & 20 & $\sim 6600$ & 0.168 & 16.5
\end{tabular}

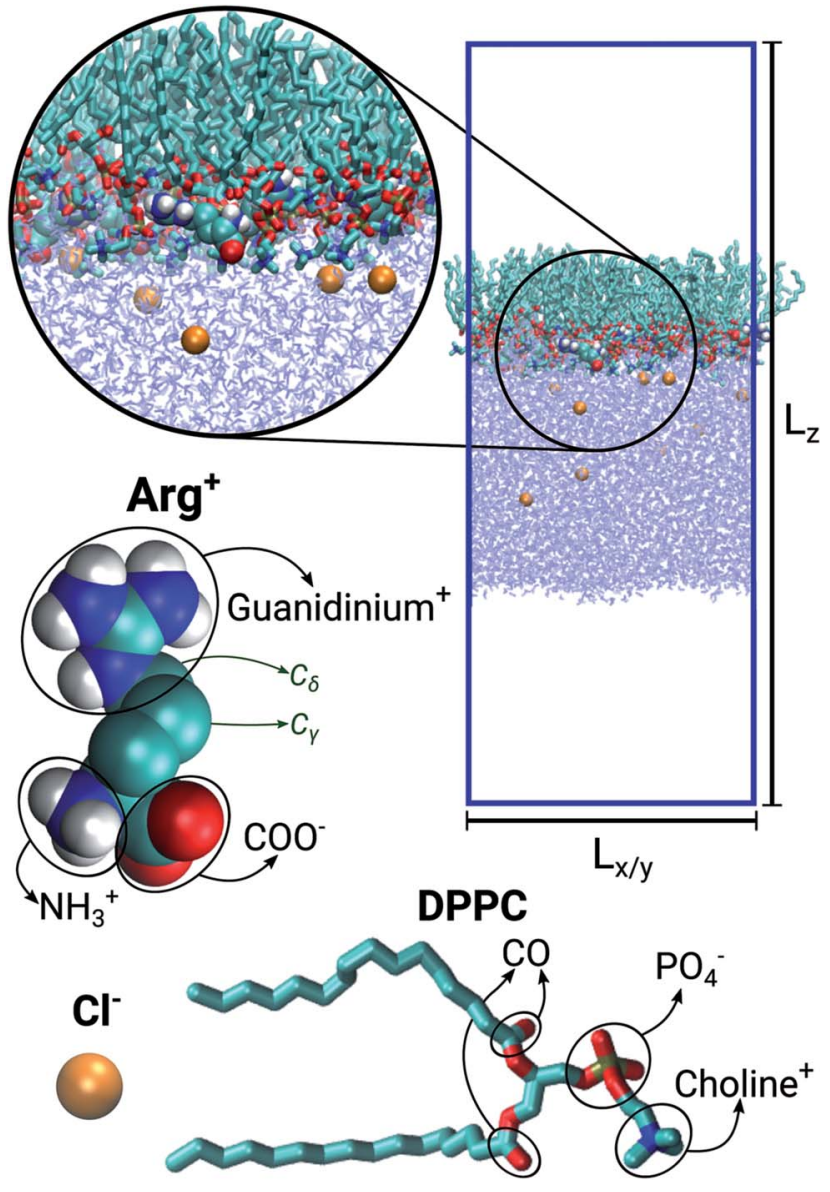

Fig. 1 Representative snapshot of the DPPC : ARG (64:10) system. $L_{z}$ and $L_{x}=L_{y}$ indicate the dimensions of the simulation box. Molecular models for chloride, arginine and DPPC.

dipole correction ${ }^{55}$ term was used in order to mitigate the effects of the permanent dipole moment of our asymmetric monolayerwater set-up. Simulations were carried out on the $N \gamma P_{z} T$ ensemble, where $N$ is the number of particles, $T=310 \mathrm{~K}$ is the temperature, $P_{z}=1$ bar is the normal component of the pressure tensor, and $\gamma$ is the surface tension. The Berendsen algorithm was used for the thermostat and barostat, ${ }^{56}$ with time constants of 0.1 and $1.0 \mathrm{ps}$ respectively. Water bond angles and lengths were constrained with the SETTLE ${ }^{57}$ algorithm, while all the other chemical bonds were constrained with LINCS. ${ }^{58}$ All calculations were run with the GROMACS 4.5 .5 code, ${ }^{59}$ using an integration time step of 2 fs and a neighbours-list update frequency of $10 \mathrm{MD}$ steps. For the calculation of compression isotherms (Section 3.1), ten different surface tensions were imposed. In the absence of dissolved $\mathrm{Arg}^{+}$, each system was equilibrated for $120 \mathrm{~ns}$ and production runs were henceforth extended for another $50 \mathrm{~ns}$. To the final configuration of these simulations, amino-acids, counter-ions and more water, were added to the sub-phase. Systems were then equilibrated for 150 ns and productions runs extended up to at least $250 \mathrm{~ns}$, but in a few cases reached 350 ns. Long simulation times were required to ensure proper equilibration of the interfacial area, as properly discussed in ref. 25 . 
The free energy profiles reported in Section 3.2 were computed by Umbrella Sampling (US). The reaction coordinate was the $Z$-distance, $d$, between the centre of mass of the monolayer and the centre of mass of one $\mathrm{Arg}^{+}$molecule. 27 US windows, defined by harmonic restraining potentials, uniformly sampled the reaction coordinate between 0 and $3.5 \mathrm{~nm}$. Force-constants ranged from 500 to $3000 \mathrm{~kJ}\left(\mathrm{~mol}^{-1}\right.$ $\AA^{-2}$ ), with the largest values used to sample the penetration of the amino-acid into the monolayer's hydrophobic region. Each US simulation was initiated from the final state of one of the unbiased trajectories used for the calculation of the compression isotherm. After applying the restraining potential to one of the amino-acids, the system was equilibrated for $5 \mathrm{~ns}$, and the simulation was subsequently extended up to 55 ns. Free energy profiles were recovered with the Weighted Histogram Analysis Method (WHAM). ${ }^{60}$

\section{Results and discussion}

\subsection{Pressure/area isotherm and surface potential}

In a typical Langmuir trough experiment the surface pressure, $\Pi$, is defined as the reduction in the surface tension of the aqueous sub-phase, $\gamma_{\mathrm{sp}}$, due to the presence of the monolayer:

$$
\Pi=\gamma_{\mathrm{sp}}-\gamma_{\mathrm{m} / \mathrm{sp}}
$$

where $\gamma_{\mathrm{m} / \mathrm{sp}}$ is the surface tension of the monolayer/aqueousphase system. At constant temperature, $\Pi$ is a function of the area per lipid, $A$, and the $\Pi-A$ relationship is called the surfacepressure/area isotherm, or simply the compression isotherm. Solutes added to the sub-phase can induce measurable changes on the isotherm, depending on how they bind to the lipid molecules and on the way they affect lateral packing. For example, solutes that remain adsorbed on the aqueous side of the interface or that are projected on top of the monolayer, affect the $\Pi-A$ curves differently to those which are partially or fully incorporated between the lipids.

Fig. 2 shows the compression isotherms for DPPC, with and without arginine dissolved in the water slab. Overall, the two isotherms display the signatures of the $2 \mathrm{D}$ phases observed in experiments and simulations of DPPC on pure water. ${ }^{25,32,61,62}$ At $310 \mathrm{~K}$ and molecular areas larger than $0.52 \mathrm{~nm}^{2}$, lipids are in the liquid expanded-phase (LE). Below $0.48 \mathrm{~nm}^{2}$ the monolayer is in the liquid-condensed phase (LC). The region in between, delimited by the cyan vertical lines in Fig. 2, corresponds to the LC-LE coexistence region. As a point of comparison, it is worth noticing that in the absence of $\mathrm{Arg}^{+}$our results closely match the isotherms reported by Mohammad-Aghaie et al. ${ }^{25}$ that were computed with the same combination of force-fields but from somewhat shorter MD trajectories.

It is clear from Fig. 2 that the addition of arginine to the sub-phase has little effect on the overall shape of the isotherm, except for a noticeable upward shift on the surface-pressure at coexistence (horizontal arrow in Fig. 2), and much larger area fluctuations within the LE region. As we will see later, these increasing fluctuations, which translate into larger uncertainties on the estimated mean areas per lipid, are the

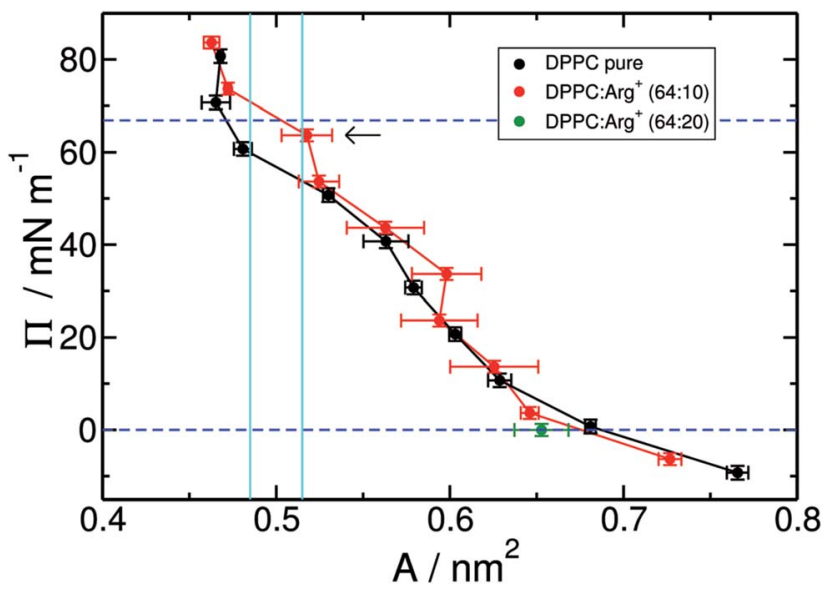

Fig. 2 Surface-pressure/area isotherms at $310 \mathrm{~K}$ for a DPPC monolayer on pure water (black points), and on an aqueous solution of $\mathrm{ArgCl}$ with a DPPC : $\mathrm{Arg}^{+}$ratio of $64: 10$ (red points). The green dot corresponds to a DPPC : $\mathrm{Arg}^{+}$ratio of $64: 20$. The region of coexistence between the liquid-expanded and liquid-condensed phases is highlighted by the cyan vertical lines.

result of the much more sluggish dynamics of the DPPC molecules in the presence of amino-acids. Notice, in particular, that within the LE phase one of the red points $(A \sim 0.6$ $\mathrm{nm}^{2}-\Pi \sim 30 \mathrm{mN} \mathrm{m}^{-1}$ ) seems to lie outside the general trend of the curve. This is most likely due to noise, as the shift from the neighbouring black curve is almost within the error bar of the red point.

The overall similarity between the isotherms could imply that the charged solutes interact very little with the lipid surface, or that they adsorb on the monolayer without greatly altering the lateral packing of DPPC. Visualisation of the simulation trajectories, and a careful analysis of the in-plane $(X-Y)$ radial distribution functions confirms that: (i) $\mathrm{Arg}^{+}$ molecules adsorb and interact specifically with the hydrophilic head of DPPC, without ever leaving the lipid/water interface during the course of 350 ns-long simulations; (ii) $\mathrm{Arg}^{++}$s remain entirely on the aqueous side of the interface, as illustrated in Fig. 3, but diffuse on the $X-Y$ plane; (iii) chlorides disperse in water forming a cloud of counter-ions; (iv) arginines do not modify in a significant way the $X-Y$ radial distribution function between the alkyl chains, nor the ordering of the lipids' head groups (see Fig. S1 and S2 of ESI $\dagger$ ). In addition, one extra simulation performed within the LE phase with twice the amount of dissolved $\mathrm{Arg}^{+}(0.168 \mathrm{M})$, still showed $100 \%$ of amino-acid adsorption (right panels in Fig. 3) without a significant impact on the resulting area per lipid (green dot in Fig. 2). Incidentally, atomistic simulations have shown that a large concentration of adsorbed $\mathrm{Arg}^{+}$has almost no effect on the area per lipid of a zwitterionic DMPC bilayer. ${ }^{63}$

The surface potential of a Langmuir layer is a measurable property that can be exploited to probe the binding of molecules to the lipid film. ${ }^{2}$ It is defined as the change in electrostatic potential, $\Delta \phi$, that occurs at a point immediately above the subphase, when the monolayer is inserted at the vacuum/liquid interface: 


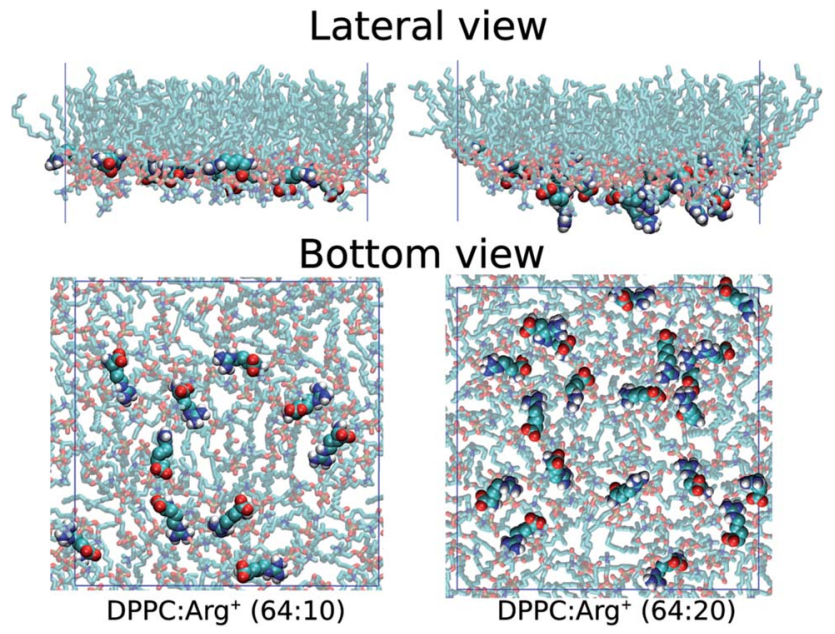

Fig. 3 Lateral and bottom views of instantaneous configurations of the $64: 10$ (left) and $64: 20$ (right) DPPC : Arg $^{+}$systems. Only DPPC and $\mathrm{Arg}^{+}$molecules are shown.

$$
\Delta \phi=\phi_{\mathrm{air} / \mathrm{m} / \mathrm{sp}}-\phi_{\mathrm{air} / \mathrm{sp}}
$$

In this equation, $\phi_{\mathrm{air} / \mathrm{m} / \mathrm{sp}}$ and $\phi_{\mathrm{air} / \mathrm{sp}}$ represent the electrostatic potential above the surface in the presence and absence of the monolayer. These quantities can be readily computed from simulations by double integration of the total charge density perpendicular to the monolayer plane, $q(z)$, according to the following equation: ${ }^{27}$

$$
\phi(z)=-\frac{1}{\varepsilon_{0}} \int_{-\infty}^{z} \mathrm{~d} z^{\prime} \int_{-\infty}^{z^{\prime}} q\left(z^{\prime \prime}\right) \mathrm{d} z^{\prime \prime},
$$

where $\varepsilon_{0}$ is the permittivity of vacuum. In practice, $q(z)$ is computed independently for the sub-phase and the LM system, and integrations are performed from a point at the centre of the vacuum region $(-\infty)$, where the electrostatic potential is referenced to zero, up to the centre of the water slab.

Our results for the DPPC monolayer on pure water, and the 64:10 and 64:20 DPPC: $\mathrm{Arg}^{+}$systems, are presented in Fig. 4. These particular data were computed within the LE phase, at $\Pi \sim 1 \mathrm{mN} \mathrm{m}^{-1}$, but similar trends are observed at other compression points. The surface potential for DPPC on pure water predicted by our simulations $\left(\Delta \phi_{\mathrm{DPPC}}=0.13 \mathrm{~V}\right)$ is slightly lower than the experimental values, that range from $0.20 \mathrm{~V}$ to $0.45 \mathrm{~V} .^{61,64-66}$ The reason for such discrepancy may well be attributed to the lack of electronic polarisability in our water model, ${ }^{27}$ an effect that if included in the force-field, would render almost unfeasible the execution of the long simulations required to equilibrate the area per lipid in the presence $\mathrm{Arg}^{+}$. More importantly, Fig. 4 shows that $\Delta \phi$ increases significantly with the amount of arginine added to the sub-phase, due to the accumulation of positive charge at the interface. This observation suggests that surface potential measurements should be more suitable than compression isotherms to determine the degree of binding of positively charged amino-acids to zwitterionic monolayers.

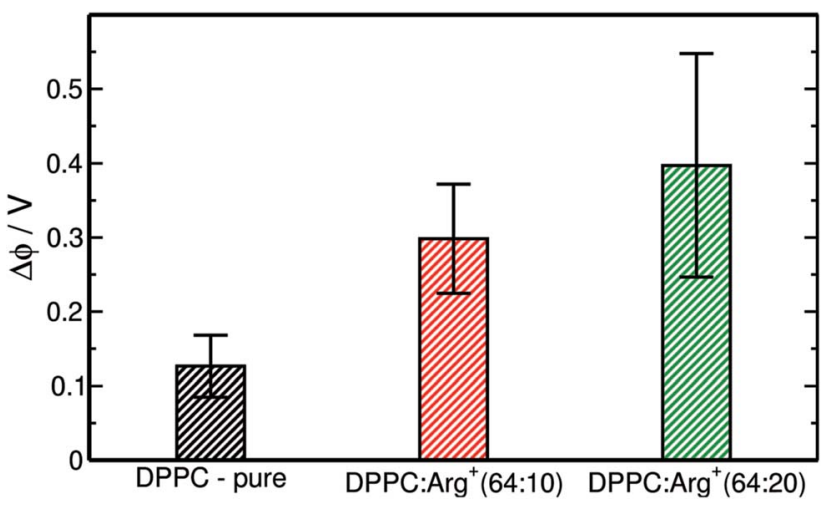

Fig. 4 Surface potential of the DPPC monolayer on pure water (black), and the $64: 10$ (red) and $64: 20$ (green) DPPC : Arg $^{+}$systems, at $\Pi \sim 1$ $\mathrm{mN} \mathrm{m}^{-1}$. The potential values are: $\Delta \phi_{\mathrm{DPPC}}=0.13 \pm 0.04 \mathrm{~V}, \Delta \phi_{64: 10}=$ $0.30 \pm 0.07 \mathrm{~V}, \Delta \phi_{64: 20}=0.40 \pm 0.15 \mathrm{~V}$.

\subsection{Energetics of arginine adsorption on DPPC}

Insight into the bonding of $\mathrm{Arg}^{+}$to the lipid phase was gained by computing the adsorption free energy profile for a single aminoacid molecule approaching the monolayer. Two simulation conditions were considered: the monolayer supported on pure water, and the $64: 20 \mathrm{DPPC}: \mathrm{Arg}^{+}$system of Fig. 2, where 20 amino-acids were pre-adsorbed on the interface. The latter system allowed for the assessment of adsorbate concentration on the binding free energy. Both systems were prepared and maintained in the LE phase during the umbrella sampling simulations, by applying a lateral pressure of $10 \mathrm{mN} \mathrm{m}^{-1}$.

The resulting free energy profiles are presented in the upper panel of Fig. 5. They both show a deep minimum near the plane defined by the $\mathrm{PO}_{4}{ }^{-}$groups of DPPC (marked with the orange vertical line), indicating that adsorption is highly favourable, and explaining why the amino-acids never detach from the interface during the course of the unbiased MD trajectories reported in the previous section. The computed adsorption energies are $-43.8 \mathrm{~kJ} \mathrm{~mol}^{-1}$ for DPPC on pure water, and -22.2 $\mathrm{kJ} \mathrm{mol}^{-1}$ for the highly concentrated $64: 20$ system, with minima located at 0.58 and $0.84 \mathrm{~nm}$ respectively. The concentration dependence of the binding energy can be ascribed to a combination of electrostatic repulsion between the approaching $\mathrm{Arg}^{+}$and those already present at the interface, and the reduced availability of binding sites when the monolayer is partially covered with amino-acids. As discussed below, $\mathrm{Arg}^{+}$interacts specifically with the $\mathrm{PO}_{4}{ }^{-}$and $\mathrm{CO}$ groups of DPPC. It is also noticeable that electrostatic repulsion with the pre-adsorbed molecules introduces a small activation barrier of around $3.3 \mathrm{~kJ} \mathrm{~mol}^{-1}$.

As with any ion-transfer reaction, ${ }^{67}$ the number of water molecules in the first solvation shell of $\mathrm{Arg}^{+}$changes during the adsorption process. This was quantified by counting the number of water molecules within a cut-off distance of $0.9 \mathrm{~nm}$, computed from the mid point between atoms $\mathrm{C}_{\gamma}$ and $\mathrm{C}_{\delta}$ of the amino-acid (see Fig. 1). Such combination of cut-off and reference point guarantees the inclusion of the water molecules associated to all the functional groups of $\mathrm{Arg}^{+}$. In the bulk of the 

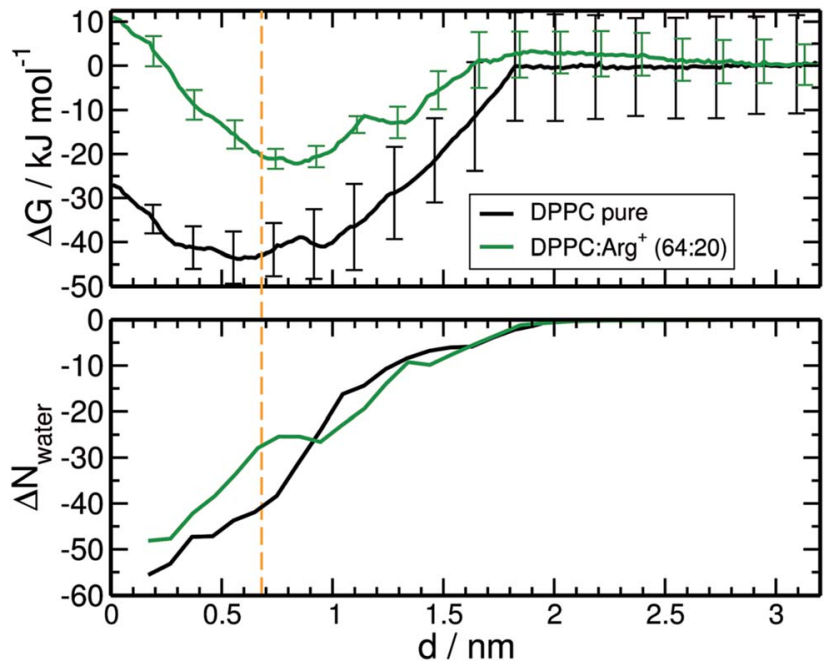

Fig. 5 Top panel. Free energy profiles for the adsorption of $\mathrm{Arg}^{+}$on the monolayer supported on pure water (black curve), and on a monolayer with 20 pre-adsorbed amino-acids (DPPC : $\operatorname{Arg}^{+} 64: 20$ system, green curve). Bottom panel: changes in the number of water molecules solvating $\mathrm{Arg}^{+}$, within a cut-off of $0.9 \mathrm{~nm}$. The orange vertical line indicates the position of the plane defined by the $\mathrm{PO}_{4}{ }^{-}$ functional groups of DPPC.

sub-phase, the so defined arginine solvation shell contains 64 molecules, no matter the number of pre-adsorbed arginines or counter-ions. As the amino-acid approaches the monolayer it loses water of solvation, but to a certain extent that depends on the composition of the system. This can be seen from the bottom panel of Fig. 5, that shows the change in the number of solvating water molecules as a function of the distance to the monolayer. As the approaching $\mathrm{Arg}^{+}$reaches the plane of the $\mathrm{PO}_{4}{ }^{-}$groups, i.e. near the minimum of the free-energy curve, it retains much more water when other amino-acids are already present at the interface $(\Delta N \sim-27 v s . \Delta N \sim-41)$. This happens because, at high interfacial concentrations, arginines tend to align vertically with respect to the monolayer plane, retaining thus a larger fraction of solvation shell upon adsorption (right panels Fig. 3). At low concentrations they tend to flatten on the interface (left panels Fig. 3) and keep a smaller solvation shell.

Specific intermolecular contacts between the most relevant functional groups of $\mathrm{Arg}^{+}$and DPPC (Fig. 1), were identified by analysing mutual coordination numbers (computed up to the first minimum of the corresponding radial distribution functions) and the average number of hydrogen bonds. The results are summarised in Table 2. Each horizontal entry has been split into two rows. The upper ones correspond to the $64: 10$ DPPC : $\mathrm{Arg}^{+}$system, and the bottom ones to the $64: 20$ concentration.

$\mathrm{NH}_{3}{ }^{+}$and guanidinium ${ }^{+}$form close and directional contacts with the $\mathrm{PO}_{4}{ }^{-}$and $\mathrm{CO}$ moieties of DPPC, with populations determined by the surface concentration of amino-acids. As stated before, at high concentration, $\mathrm{Arg}^{+}$molecules align vertically with respect to the interface. This is reflected by the decrease in $\mathrm{NH}_{3}{ }^{+}-\mathrm{CO}$ coordination and $\mathrm{H}$-bond numbers, and the concomitant increase in $\mathrm{PO}_{4}{ }^{-}-\mathrm{NH}_{3}{ }^{+}$contacts. At the same
Table 2 Average number of hydrogen bonds and coordination numbers between the functional groups of $\mathrm{Arg}^{+}$and DPPC. Each horizontal entry has been split into two rows. The upper rows correspond to the $64: 10$ DPPC : $\mathrm{Arg}^{+}$system, and the bottom rows to the $64: 20$ concentration

\begin{tabular}{llllllll}
\hline & \multicolumn{2}{l}{ H-Bonds } & & \multicolumn{2}{l}{ Coord. number } & \\
\cline { 2 - 3 } \cline { 5 - 6 } $\mathrm{Arg}^{+} / \mathrm{DPPC}$ & $\mathrm{PO}_{4}{ }^{-}$ & $\mathrm{CO}$ & & $\mathrm{PO}_{4}{ }^{-}$ & $\mathrm{CO}$ & Choline $^{+}$ \\
\hline $\mathrm{NH}_{3}{ }^{+}$ & $1.2 \pm 0.2$ & $1.6 \pm 0.2$ & 1.31 & 1.79 & - \\
& $1.5 \pm 0.1$ & $1.1 \pm 0.1$ & 1.66 & 0.36 & \\
Guanidinium $^{+}$ & $1.5 \pm 0.3$ & $1.2 \pm 0.2$ & 1.78 & 1.87 & - \\
$\mathrm{COO}^{-}$ & $1.3 \pm 0.2$ & $0.8 \pm 0.2$ & 1.46 & 1.38 & \\
Total & - & - & - & - & 2.10 \\
& $5.6 \pm 0.4$ & & 8.85 & & \\
& $4.8 \pm 0.3$ & & 6.70 & & \\
\hline
\end{tabular}

time, the guanidinium ${ }^{+}$groups lose coordination and binding to both $\mathrm{PO}_{4}^{-}$and $\mathrm{CO}$, as the carboxylate moiety $\left(\mathrm{COO}^{-}\right)$gets farther away from the choline moiety of DPPC. In support of our findings, a number of spectroscopic measurements performed on vesicles and Langmuir monolayers, ${ }^{68-71}$ have shown clear changes on the stretching mode of the $\mathrm{CO}$ and $\mathrm{PO}_{4}{ }^{-}$groups of several phospholipids upon adsorption of positively charged molecules, including amino-acids. These observations have been interpreted in terms of changes of hydration and hydrogen-bonding patterns. Also, previous simulations of $\mathrm{Arg}^{+}$ in contact with a DMPC bilayer have demonstrated that the guanidinium ${ }^{+}$groups interact with the $\mathrm{PO}_{4}{ }^{-}$, displacing at most two water molecules per amino-acid. ${ }^{63}$

\subsection{Arginine modulates the lateral mobility of DPPC}

As discussed in the previous sections, arginine binds tightly to the lipids' head groups while retaining lateral mobility. This may affect the diffusivity of the lipids in varying extends, depending on the contact dynamics between the amino-acids and the DPPC molecules. Assessing the impact of adsorbates on the diffusion coefficient of the lipids is important, not only to understand how the sub-phase composition affects the molecular dynamics of the monolayer, but also because transport coefficients can be measured experimentally. ${ }^{72}$ Diffusion coefficients may then provide an alternative, or complementary, way of sensing amino-acid-lipid interactions in LM set-ups.

The lateral mean-square displacement of DPPC was computed at different lateral pressures for each of the systems described in Table 1. As expected, within the LC phase the lipids' dynamics are very sluggish, making the evaluation of diffusion coefficients from simulations too uncertain. Within the LE phase, the DPPC molecules move more readily and diffusion coefficients, $D$, could be reliably computed using the Einstein relation. ${ }^{73}$ Fig. 6 collects the results obtained at a lateral pressure of $\sim 1 \mathrm{mN} \mathrm{m}^{-1}$. It is clear that the presence of $\mathrm{Arg}^{+}$in the sub-phase has a drastic effect on the lateral mobility of DPPC. For the monolayer supported on pure water $D=1.3 \times$ $10^{-7} \mathrm{~cm}^{2} \mathrm{~s}^{-1}$, while the experimental values for DPPC in the LE phase range from $1.0 \times 10^{-7}$ to $5 \times 10^{-7} \mathrm{~cm}^{2} \mathrm{~s}^{-1} \cdot{ }^{72,74-76} \mathrm{Arg}^{+}$ 


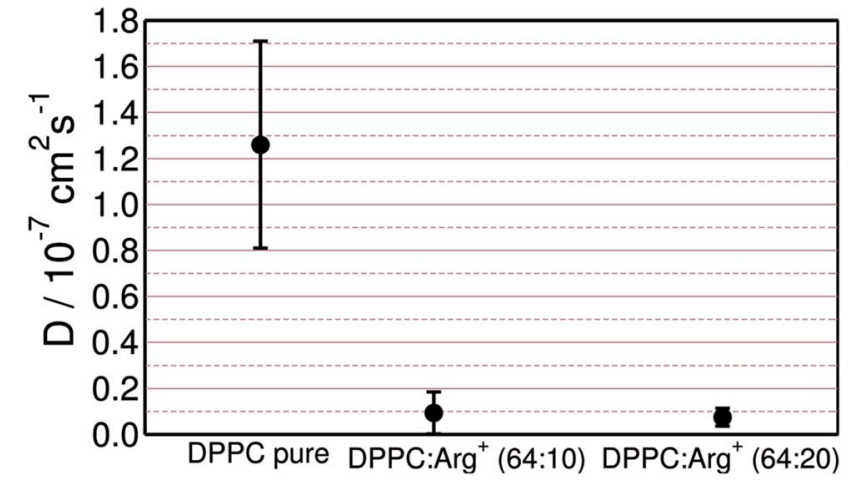

Fig. 6 DPPC self-diffusion coefficients computed for the monolayer on pure water, and the $64: 10$ and $64: 20$ DPPC : $\mathrm{Arg}^{+}$systems, at $\Pi$ $\sim 1 \mathrm{mN} \mathrm{m}^{-1}$.

lowers the diffusivity of the lipids by one order of magnitude, leading to $D \sim 1.0 \times 10^{-8} \mathrm{~cm}^{2} \mathrm{~s}^{-1}$, independently of the aminoacid concentration.

The reason for the decrease in self-diffusion coefficients is the formation of small DPPC clusters held together by a single $\mathrm{Arg}^{+}$molecule. As discussed in Section 3.2, the molecular contacts that confer stability to the binding of arginine to DPPC occur between the cationic groups of the amino-acid and the $\mathrm{PO}_{4}{ }^{-}$and $\mathrm{CO}$ groups. However, the mere existence of such contacts does not necessarily explain the slowdown of the lipids mobility, as contacts could break and form quickly.

In order to gain insight into the dynamical behaviour of the small DPPC clusters, we computed a "cage correlation function" $n(t)$, as described in ref. 77. $n(t)$ monitors the rate at which DPPC molecules interchange their closest partners. This is done by identifying all the neighbours of each lipid within a cut-off distance $R_{\mathrm{c}}$ at time $t_{\mathrm{o}}=0$, and computing the average number of molecules still belonging to the same coordination shell at any later time $t$. In our simulations, $R_{\mathrm{c}}=0.75 \mathrm{~nm}$, which corresponds to the first minimum of the radial distribution

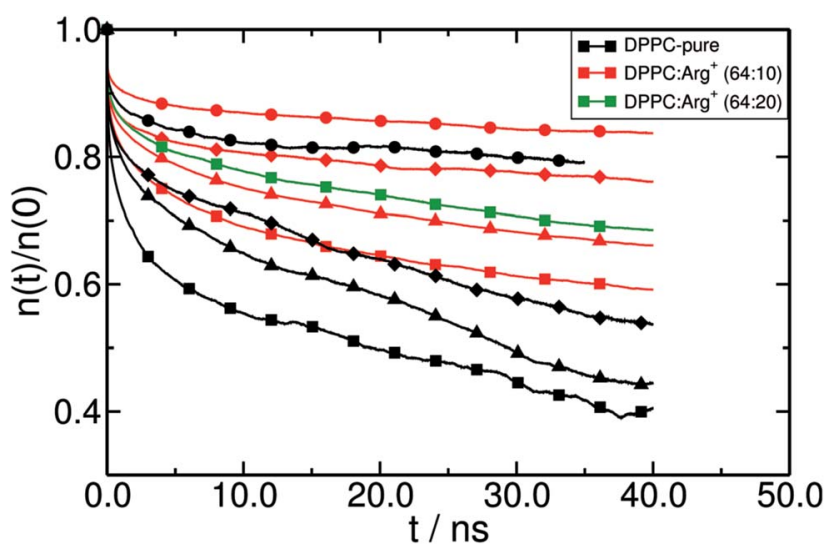

Fig. 7 Cage correlation function, $n(t)$, for different systems at various surface pressures. Black lines $=$ DPPC monolayer on pure water, red lines $=64: 10$ DPPC $: \mathrm{Arg}^{+}$system, green line $=64: 20 \mathrm{DPPC}: \mathrm{Arg}^{+}$ system. Circles: $\Pi \sim 70 \mathrm{mN} \mathrm{m}^{-1}$; diamonds: $\Pi \sim 50 \mathrm{mN} \mathrm{m}^{-1}$, triangles: $\Pi \sim 20 \mathrm{mN} \mathrm{m}^{-1}$, and squares: $\Pi \sim 1 \mathrm{mN} \mathrm{m}^{-1}$. function between the $\mathrm{PO}_{4}{ }^{-}$groups. The results are presented in Fig. 7. Clearly, $\mathrm{Arg}^{+}$reduces the rate of first-neighbours exchange, which translates into longer lifetimes of the DPPC aggregates that the amino-acids help keeping together. The effect can be detected at all surface pressures, but is systematically more evident within the LE phase. Also, note that increasing the concentration of $\mathrm{Arg}^{+}$increases the lifetime of the lipid's first coordination shell (compare the green and red lines with squares, in Fig. 7).

\section{Conclusions}

We have investigated by means of molecular dynamics simulations how and to what extent arginine affects the compression isotherm, the interfacial potential, and the lateral mobility of the lipids in a Langmuir monolayer. Free energy calculations show that $\mathrm{Arg}^{+}$binds strongly to the DPPC hydrophilic surface, with adsorption energies of $-43.8 \mathrm{~kJ} \mathrm{~mol}^{-1}$ for the bare interface, and $-22.2 \mathrm{~kJ} \mathrm{~mol}^{-1}$ in the case of the monolayer precovered with $\mathrm{Arg}^{+}$(64:20 DPPC: $\mathrm{Arg}^{+}$ratio). The large binding energy to the bare surface can be attributed to hydrogen bonding between the charged moieties of $\mathrm{Arg}^{+}$and the $\mathrm{PO}_{4}^{-}$ and CO groups of DPPC, which compensates for changes in the levels of hydration of the lipids and the amino-acid upon adsorption. As the concentration of pre-adsorbed amino-acids increases, arginine molecules align more vertically with respect to the plane of the monolayer, and electrostatic repulsion lowers the binding energy and even introduces a small activation barrier $\left(\sim 3.3 \mathrm{~kJ} \mathrm{~mol}^{-1}\right)$ on the adsorption free-energy profile.

Apart from a small upward shift on the surface-pressure at coexistence, $\mathrm{Arg}^{+}$does not greatly affect the compression isotherm of DPPC at $310 \mathrm{~K}$. This happens because, both in the LC and LE phase, the amino-acids remain on the aqueous side of the interface without showing significant insertion between the lipids. In fact, at the highest surface concentration explored, a population of arginine molecules hang vertically from the monolayer plane and into the aqueous phase. However, the accumulation of interfacial charge has a significant effect on the interfacial potential, that goes from $0.13 \mathrm{~V}$ for the bare lipid/ water interface, to $0.40 \mathrm{~V}$ when the DPPC : $\mathrm{Arg}^{+}$ratio is $64: 20$.

The dynamical properties of the lipids are strongly affected by the presence of $\mathrm{Arg}^{+}$in the sub-phase. At an even moderate concentration of amino-acid (64:10 DPPC: $\mathrm{Arg}^{+}$), the selfdiffusion coefficient of DPPC decreases in one order of magnitude with respect to that of the monolayer supported on pure water. This is due to the formation of transient, but long-lived, DPPC-Arg ${ }^{+}$complexes that increase the average caging time of the lipids. In these complexes, each arginine molecule connects to one, or bridges together two or three lipid molecules.

Ultimately, we have shown that the strong adsorption of $\mathrm{Arg}^{+}$ on DPPC has little impact on the lateral mechanical properties of the monolayer, but has a significant and measurable effect on its electrical and dynamical properties. We believe that our results provide new molecular-level insight into the interfacial behaviour of self-assembled lipid structures doped with highly hydrophilic amino-acids. 


\section{Acknowledgements}

The authors thank CONICET, SECTyP-UNCUYO, and FONCyT (PICT-2012-2759) for research funding. We are also grateful to the EU Commission Marie Curie RISE "ENACT" Programme (643998), and SNCAD-MinCyT for computer time allocation in the clusters Mendieta (CCAD-UNC) and Toko (UNCUYO).

\section{References}

1 Y.-H. M. Chan and S. G. Boxer, Curr. Opin. Chem. Biol., 2007, 11, 581-587.

2 H. Brockman, Curr. Opin. Chem. Biol., 1999, 9, 438-443.

3 V. M. Kaganer, H. Möhwald and P. Dutta, Rev. Mod. Phys., 1999, 71, 779-819.

4 G. Brezesinski and H. Möhwald, Adv. Colloid Interface Sci., 2003, 100, 563-584.

5 R. Maget-Dana, Biochim. Biophys. Acta, Biomembr., 1999, 1462, 109-140.

6 E. Maltseva and G. Brezesinski, ChemPhysChem, 2004, 5, 1185-1190.

7 A. Won and A. Ianoul, Biochim. Biophys. Acta, Biomembr., 2009, 1788, 2277-2283.

8 L. Caseli, D. C. Masui, R. P. Furriel, F. A. Leone, M. E. Zaniquelli, J. Orbulescu and R. M. Leblanc, J. Colloid Interface Sci., 2008, 320, 476-482.

9 M. M. Domon, M. N. Nasir, S. Pikula and F. Besson, J. Colloid Interface Sci., 2013, 403, 99-104.

10 Z. Kozarac, B. Ćosović, D. Möbius and M. Dobrić, J. Colloid Interface Sci., 2000, 226, 210-217.

11 F. J. Pavinatto, A. Pavinatto, L. Caseli, D. S. dos Santos, T. M. Nobre, M. E. Zaniquelli and O. N. Oliveira, Biomacromolecules, 2007, 8, 1633-1640.

12 M. N. Antipina, I. Schulze, M. Heinze, B. Dobner, A. Langner and G. Brezesinski, ChemPhysChem, 2009, 10, 2471-2479.

13 M. Dittrich, M. Bottcher, J. S. L. Oliveira, B. Dobner, H. Mohwald and G. Brezesinski, Soft Matter, 2011, 7, 10162-10173.

14 S. A. Kane and S. D. Floyd, Phys. Rev. E: Stat. Phys., Plasmas, Fluids, Relat. Interdiscip. Top., 2000, 62, 8400-8408.

15 S.-Y. Choi, S.-G. Oh and J.-S. Lee, Colloids Surf., B, 2001, 20, 239-244.

16 M. Gagoś, J. Gabrielska, M. D. Serra and W. I. Gruszecki, Mol. Membr. Biol., 2005, 22, 433-442.

17 K. Hacc-Wydro, J. Kapusta, A. Jagoda, P. Wydro and P. Dynarowicz-Łątka, Chem. Phys. Lipids, 2007, 150, 125-135.

18 A. Karewicz, D. Bielska, B. Gzyl-Malcher, M. Kepczynski, R. Lach and M. Nowakowska, Colloids Surf., B, 2011, 88, 231-239.

19 S.-S. Feng, K. Gong and J. Chew, Langmuir, 2002, 18, 40614070.

20 A. T. Jones and E. J. Sayers, J. Controlled Release, 2012, 161, 582-591.

21 P. Ahlstrom and H. J. C. Berendsen, J. Phys. Chem., 1993, 97, 13691-13702.

22 Y. N. Kaznessis, S. Kim and R. G. Larson, Biophys. J., 2002, 82, 1731-1742.
23 S. K. Kandasamy and R. G. Larson, Biophys. J., 2005, 88, 1577-1592.

24 M. Pickholz, O. N. Oliveira and M. S. Skaf, Biophys. Chem., 2007, 125, 425-434.

25 D. Mohammad-Aghaie, E. Macé, C. A. Sennoga, J. M. Seddon and F. Bresme, J. Phys. Chem. B, 2010, 114, 1325-1335.

26 H.-Z. Gang, J.-F. Liu and B.-Z. Mu, J. Phys. Chem. B, 2011, 115, 12770-12777.

27 T. R. Lucas, B. A. Bauer, J. E. Davis and S. Patel, J. Comput. Chem., 2012, 33, 141-152.

28 M. Chen, X. Lu, X. Liu, Q. Hou, Y. Zhu and H. Zhou, Langmuir, 2014, 30, 10600-10607.

29 C.-P. Kong, E. Peters, Q.-C. Zheng, H.-X. Zhang, et al., Phys. Chem. Chem. Phys., 2014, 16, 9634-9642.

30 M. Gorczyca, B. Korchowiec, J. Korchowiec, S. Trojan, J. Rubio-Magnieto, S. V. Luis and E. Rogalska, J. Phys. Chem. B, 2015, 119, 6668-6679.

31 W. Lin, A. J. Clark and F. Paesani, Langmuir, 2015, 31, 21472156.

32 S. L. Duncan and R. G. Larson, Biophys. J., 2008, 94, 29652986.

33 A. J. Kox, J. P. J. Michels and F. W. Wiegel, Nature, 1980, 287, 317-319.

34 J. Harris and S. A. Rice, J. Chem. Phys., 1988, 89, 5898-5908. 35 S. Karaborni and S. Toxvaerd, J. Chem. Phys., 1992, 96, 55055515.

36 S. O. Nielsen, C. F. Lopez, P. B. Moore, J. C. Shelley and M. L. Klein, J. Phys. Chem. B, 2003, 107, 13911-13917.

37 S. Baoukina, L. Monticelli, S. J. Marrink and D. P. Tieleman, Langmuir, 2007, 23, 12617-12623.

38 J. Giner-Casares, L. Camacho, M. Martin-Romero and J. Lopez Cascales, Langmuir, 2008, 24, 1823-1828.

39 W. Shinoda, R. DeVane and M. L. Klein, J. Phys. Chem. B, 2010, 114, 6836-6849.

40 V. Miguel, M. A. Perillo and M. A. Villarreal, Biochim. Biophys. Acta, Biomembr., 2016, 1858, 2903-2910.

41 E. Harder and B. Roux, J. Chem. Phys., 2008, 129, 234706.

42 J. Chowdhary, E. Harder, P. E. M. Lopes, L. Huang, A. D. MacKerell and B. Roux, J. Phys. Chem. B, 2013, 117, 9142-9160.

43 E. Harder, A. D. MacKerell Jr and B. Roux, J. Am. Chem. Soc., 2009, 131, 2760-2761.

44 G. Casares, J. José, L. Camacho, M. T. Martín-Romero and J. J. López Cascales, ChemPhysChem, 2008, 9, 2538-2543.

45 B. Liu, M. I. Hoopes and M. Karttunen, J. Phys. Chem. B, 2014, 118, 11723-11737.

46 G. Casares, J. José, L. Camacho, M. T. Martín-Romero and J. J. López Cascales, ChemPhysChem, 2010, 11, 2241-2247.

47 J. A. Freites, Y. Choi and D. J. Tobias, Biophys. J., 2003, 84, 2169-2180.

48 C. Laing, S. Baoukina and D. P. Tieleman, Phys. Chem. Chem. Phys., 2009, 11, 1916-1922.

49 L. Li, I. Vorobyov and T. W. Allen, J. Phys. Chem. B, 2013, 117, 11906-11920.

50 C. Schwieger and A. Blume, Biomacromolecules, 2009, 10, 2152-2161. 
51 O. Berger, O. Edholm and F. Jähnig, Biophys. J., 1997, 72, 2002.

52 J. L. Abascal and C. Vega, J. Chem. Phys., 2005, 123, 234505.

53 C. Oostenbrink, T. A. Soares, N. F. A. van der Vegt and W. F. van Gunsteren, Eur. Biophys. J., 2005, 34, 273-284.

54 U. Essmann, L. Perera, M. L. Berkowitz, T. Darden, H. Lee and L. G. Pedersen, J. Chem. Phys., 1995, 103, 8577-8593.

55 I.-C. Yeh and M. L. Berkowitz, J. Chem. Phys., 1999, 111, 3155-3162.

56 H. J. Berendsen, J. v. Postma, W. F. van Gunsteren, A. DiNola and J. Haak, J. Chem. Phys., 1984, 81, 3684-3690.

57 S. Miyamoto and P. A. Kollman, J. Comput. Chem., 1992, 13, 952-962.

58 B. Hess, H. Bekker, H. J. Berendsen, J. G. Fraaije, et al., J. Comput. Chem., 1997, 18, 1463-1472.

59 B. Hess, C. Kutzner, D. Van Der Spoel and E. Lindahl, J. Chem. Theory Comput., 2008, 4, 435-447.

60 S. Kumar, J. M. Rosenberg, D. Bouzida, R. H. Swendsen and P. A. Kollman, J. Comput. Chem., 1992, 13, 1011-1021.

61 V. L. Shapovalov, E. A. Kotova, T. I. Rokitskaya and Y. N. Antonenko, Biophys. J., 1999, 77, 299-305.

62 A. Rosengarth, A. Wintergalen, H.-J. Galla, H.-J. Hinz and V. Gerke, FEBS Lett., 1998, 438, 279-284.

63 F. E. Herrera, A. Bouchet, F. Lairion, E. A. Disalvo and S. Pantano, J. Phys. Chem. B, 2012, 116, 4476-4483.

64 H. Nakahara, S. Nakamura, H. Kawasaki and O. Shibata, Colloids Surf., B, 2005, 41, 285-298.
65 A. M. Farnoud and J. Fiegel, J. Phys. Chem. B, 2013, 117, 12124-12134.

66 D. S. Alvares, M. L. Fanani, J. R. Neto and N. Wilke, Biochim. Biophys. Acta, Biomembr., 2016, 1858, 393-402.

67 W. Schmickler, Electrochim. Acta, 1996, 41, 2329-2338.

68 W. Hübner and A. Blume, Chem. Phys. Lipids, 1998, 96, 99123.

69 A. Hädicke and A. Blume, Biochim. Biophys. Acta, Biomembr., 2016, 1858, 1196-1206.

70 V. P. Geraldo, F. J. Pavinatto, T. M. Nobre, L. Caseli and O. N. Oliveira, Chem. Phys. Lett., 2013, 559, 99-106.

71 M. Dyck, A. Kerth, A. Blume and M. Lösche, J. Phys. Chem. B, 2006, 110, 22152-22159.

72 F. Caruso, F. Grieser, P. J. Thistlethwaite and M. Almgren, Biophys. J., 1993, 65, 2493-2503.

73 M. P. Allen and D. J. Tildesley, Computer simulation of liquids, Oxford university press, 1989.

74 R. Peters and K. Beck, Proc. Natl. Acad. Sci. U. S. A., 1983, 80, 7183-7187.

75 M. Gudmand, M. Fidorra, T. Bjørnholm and T. Heimburg, Biophys. J., 2009, 96, 4598-4609.

76 D.-W. Jeong, K. Kim, S. Lee, M. C. Choi and S. Q. Choi, Langmuir, 2014, 30, 14369-14374.

77 M. G. Del Pópolo, C. L. Mullan, J. D. Holbrey, C. Hardacre and P. Ballone, J. Am. Chem. Soc., 2008, 130, 7032-7041. 